\title{
A Strategy for Self-Organized Coordinated Motion of a Swarm of Minimalist Robots
}

\author{
Ataollah Ramezan Shirazi, Yaochu Jin, Fellow, IEEE
}

\begin{abstract}
Minimalist robots are functionally highly restricted but well suited for swarm robotic applications because of their low costs and small sizes. Connectivity maintenance and collision avoidance are challenging in minimalist swarm robotic systems due to a short communication range and the lack of positional and directional sensing. In this paper, we introduce a selforganizing control strategy for collective flocking of a swarm of minimalist robots with an aim to improve swarm connectivity and to reduce the chance of collision between robots. Based on the relative positional information built up via collaborations, each robot determines a collision-free operational polygon. This scheduling scheme coordinates the motion of the robots by dividing them into one group of immobile and one group of mobile robots, such that each mobile robot is surrounded by immobile robots serving as beacons. In addition, we introduce a cohesive force into motion planning, which has been shown to play an important role in maintaining a swarm during flocking. A new quantitative metric is introduced for measuring the connectivity of a swarm of agents with local communications, thereby evaluating the performance of the proposed control scheme. We run extensive simulations using simulated Kilobts to examine the influence of different sources of noise and the size of swarms on the connectivity in the swarm and the speed of flocking. Finally, we implement the proposed algorithm on a swarm of real Kilobots to compare the flocking performance with and without the proposed control strategy for coordinated and collective motion.
\end{abstract}

Index Terms-Swarm Robotics, Collective Movement, Flocking, Morphogen, Self-Organizing Systems

\section{INTRODUCTION}

Emergence of collective behaviours from large number of simple agents is one of the major topics of current research in swarm robotics. Many control schemes have been developed for collaboration of robots in multi-robotic systems [1], [2]. However, most of them were confined to simulations or experiments on a small number of physical robots, mostly because the proposed algorithms require functionalities that are available on expensive robots only [3]. Such functionalities include long-range communication capability [4], vision systems [5], global localization or bearing [6]-[8], directional sensing [9], [10], digital compass [11], holonomic motion [12], motion feedback signal [13], and ability of measuring the speed and heading of near-by robots [6], [14]. Unfortunatly, it is usually not affordable to have a large number of robots having these functionalities. In addition, it is very challenging or expensive

This research was funded by the European Commission 7th Framework Program "SWARM-ORGAN" (Project No. 601062).

A. Ramezan Shirazi and Y. Jin are with the Department of Computer Science, University of Surrey, Guildford GU2 7XH, U.K. (e-mail: a.ramezanshirazi, yaochu.jin@surrey.ac.uk). (Corresponding author: Yaochu Jin) to miniaturize these functionalities by e.g., implementing them in micro/nano swarm robotic systems [15].

In general, functional simplicity of agents is essential for swarm robotic systems [16], [17]. The functionally simpler a robot is, the more affordable it will be and, the smaller its size can be. Moreover, a smaller and simpler robot consumes less energy and can potentially operate in a longer period of time. However, control of a swarm of minimalist robots is also challenging [18]. In this work, we aim to address some issues related to motion planning and connectivity maintenance of robots with highly limited sensing, communication and motion capabilities. Note that we focus on collective flocking, which is one of primitive yet important swarm behaviours.

In collective flocking, a large number of robots move toward a target, where swarm connectivity is of great importance. First, because of limited sensing ability, decision making would be inaccurate or impossible for an individual robot disconnected from the rest of the swarm [19]. Second, like in many biological systems, from which many swarm robotic systems are inspired, global collective behaviours emerge from local interactions between agents. Thus, disruption of local interactions can disable the emergence of collective behaviours. It is well known that connectivity maintenance in swarm robotic systems remains an issue even for robots that are able to self-localize themselves [20]. Therefore, it becomes even more challenging for minimalist robots, whose communication range is just few times larger than their body size and which do not have directional sensing, nor global localization [21]. Lack of directional sensing, non-holonomic motion, no motion feedback together highly increases the chance of collisions as the number of robots in the swarm increases, which has a destructive impact on motion planning.

It has been demonstrated that exchanging local information is able to compensate individual deficiencies. Nembrini and Windfield [22] compared two algorithms, called $\alpha$ and $\beta$, for connectivity maintenance of a swarm of robots without directional sensing. In both algorithms, a criterion for local connectivity is defined to enable each robot to be able to measure its level of connectivity in the swarm. Once this criterion is below a certain threshold, the robot will take a particular action to enhance its local connectivity. In $\alpha$ algorithm, this criterion is based on individual sensing data, or when there is no data exchange with others. In the $\beta$ algorithm, by contrast, extra information acquired through local communication is employed to estimate local connectivity. Here, each robot shares a list of its neighbours with its neighboring robots. The authors showed that the performance of the $\beta$ algorithm is much better than that of the $\alpha$ algorithm, since the robots are able to accurately estimate their local connectivity and 
consequently their actions for connectivity enhancement will be more timely.

Note that relative position and heading information can be achieved via cooperation in a robotic system. Cooperative positioning of locally aware multi-agent systems has received increasing attention in the last two decades. However, most developed algorithms assume the presence of either scattered globally position-aware reference points among agents [23], or directional information [24]-[26], or wheel odometry system [27]. Some of them were developed for localization of stationary agents only [28].

Recently, Cornejo and Nagpal [29] proposed an algorithm for relative localization and local coordination of a swarm of simple robots capable of measuring their distance to their close-by neighbours. In their research, similar to this work, some robots move toward the goal and the others stay immobile, providing mobile robots with positional information. However, their algorithm is computationally expensive and it requires communication proportional to the number of neighbors. At each round, a robot must send its own odometry estimations together with the relative position to its neighbors (one for each of its neighbour). Therefore, each robot in the swarm must send separate message packages with certain information specifically for each individual neighbour. Due to slow communication and small message packages, data exchange is time consuming. In addition, the authors also assumed that the robots have an inaccurate kind of odometry, which is adequate for estimation of a few previous displacements.

In this paper, we introduce a coordinated motion control algorithm for collective flocking of a swarm of minimalist robots. The proposed algorithm does not assume the need for an odometry system to estimate a robots displacement, nor the need to provide neighboring individuals with data. The proposed control strategy is self-organized and starts from identically-programmed agents with a unique ID.

In the next section, we present an algorithm for a swarm of aggregated minimalist robots to be able to localize themselves in relation to a collaboratively selected focal robots. In Section III, a mechanism is proposed to enable the robots to approximate a collision-free operational polygonal area. A collaborative motion scheme is suggested to divide robots into two groups of mobile and immobile robots with different tasks. In Section IV, we introduce a new metric for measuring the connectivity of a swarm of agents with a short communication range. Then, we report the results of extensive simulations to evaluate the performance of the algorithm. Finally, we implement the proposed algorithm in a real swarm of Kilobots, and compare the results with a situation where the localization mechanism and the motion scheduling are left off.

\section{COOPERATIVE SELF-ORGANIZED LOCALIZATION}

In awarm robotic systems, it is a common practice to localize themselves with the help of local communication with with neighboring. However, one fundamental assumption in many of these algorithms is that there are some agents, called anchor nodes, that have predetermined positions or are equipped with positioning systems like GPS. Without such anchor nodes, other robots in the swarm will not be able to function properly. However, in swarm robotics, it is assumed that global behaviours emerge from homogeneous agents such that the replacement and rearrangement of agents should not substantially influence the emergence of global behaviours. This is one of the main requirements on swarm robotic systems that is expected to enhance robustness and fault tolerance.

For mobile agents, cooperative localization is more challenging. If the calculation and communication for localization procedure are too slow to be compatible with the displacement of agents, then the estimated positions become highly inaccurate. As data exchange between agents occur periodically, there is a time interval between updating and processing messages from neighbours with broadcasting the outcomes. Displacement of robots during this time interval adds an accumulative noise to the system. One solution is to consider robots to be immobile intermittently and serve as beacons for the other mobile one [29].

Consider a swarm of robots, where each robot is able to measure its distance to the robots inside its communication range. Each robot in a swarm can be seen as a node in a graph, which is termed a communication graph in this work. Node $A$ in the graph is linked to node $B$ if node $B$ is in the communication range of node $A$. Based on the assumption that the communication range of all robots are equal, the communication graph's links are bidirectional. If a node has a three localized nodes with known distances in its neighbourhood, then it is able to calculate its own relative position using trilateration. We assume that the robots in the swarm are properly distributed so that the communication graph is connected, i.e., each node has more than two links to other nodes. In this way, positional information can propagate through the swarm by trilateration.

To establish a coordinate system for localization, we first determine the centre of coordinate system. In our previous work [30], we developed a mechanism for identification of the robot at the centre of a swarm, termed center-bot, based on artificial morphogen diffusion [7], [21]. This work does not address the aggregation process and assume that all robots are initially aggregated so that they are able to communicate with at least their immediate neighbours. The algorithm starts with the differentiation of the agents at the boundary of the swarm, termed edge-bots, based on the comparison of the number of neighboring robots of this robot's neighbours. Then, all edgebots act as a source of morphogen and a concentration gradient forms. Considering a convex shape swarm, this gradient is minimum at the centre. Hence, it is possible to identify the robot that is at the centre of the swarm by comparing the concentration level of a robot with its neighbours. Refer to [30] for more details. Once the center-bot is identified, its localization status is set to determined, and its position to $(0,0)$.

In this algorithm, the center-bot determines the centre of the coordinate system and sets up the coordinate system. First, the center-bot propagates the ID of its closest neighbour. This closest neighbour, named $\mathrm{x}$-bot, determines the $\mathrm{x}$-axes of the 
swarm coordinate system. Once $\mathrm{x}$-bot has received a message from center-bot and identified itself as the x-bot, it sets its position to $\left(d_{c x}, 0\right)$, where $d_{c x}$ is the distance between the $\mathrm{x}$ bot and center-bot. Then, it changes its localization status to determined and propagates its position.

Second, a third bot, termed $h$-bot, needs to be identified, which will determine the handedness of the coordinate system. The h-bot needs to satisfy two conditions in order to localize itself and enable its neighboring robots to estimates their position. The first condition is that it should be within the communication range of both the $\mathrm{x}$-bot and center-bot. The second condition is that it should not be close to the line passing through the $\mathrm{x}$-bot and the center-bot; otherwise, other robots may fail to properly localize themselves because of noisy measurements.

We assign the byte of the messages for communication of internal states (refer to section III-D). Each bit in the second byte represents one specific status. Once the x-bot has identified itself, it sets one dedicated bit in the status byte to label itself as the x-bot. Consequently, those robots that can communicate with the $\mathrm{x}$-bot set another bit in the status byte to indicate that "I see the $x$-bot", and send a message containing their distance from the $\mathrm{x}$-bot. Once this is done, one robot can be chosen as the h-bot by the center-bot from those that can see the $\mathrm{x}$-bot. The robot that minimize following criterion is eventually chosen as the h-bot, and its ID is propagated by the center-bot.

$$
\min _{i}\left(\left|d_{c x}-d_{i x}\right|+\left|d_{c x}-d_{c i}\right|\right) \text {, }
$$

where $d_{c x}$ and $d_{c i}$ are the distances from the center-bot to the x-bot and robot $i$, respectively, and $d_{i x}$ is the distance between robot $i$ and the x-bot, which is measured and sent by robot $i$.

Because of omni-directional sensing and the lack of global information, determination of the handedness of the coordinate system is impossible without movement. Note that at this stage, we ignore the flip ambiguity of the coordinate system. Once robots start to move, this flip ambiguity will be detected and corrected (refer to section III). Therefore, we assume that the $\mathrm{h}$-bot is on the top of the $\mathrm{x}$-axis, and the $\mathrm{h}$-bot can calculate its relative position as $\left(d_{h c} \cos (\alpha), d_{h c} \sqrt{(1-\cos (\alpha)}\right)$, where $d_{h c}$ is the distance between the h-bot and the center-bot measured by the h-bot, and $\cos (\alpha)$ is equal to:

$$
\cos (\alpha)=\frac{d_{c x}^{2}+d_{h c}^{2}-d_{h x}^{2}}{2 d_{c x} d_{h c}},
$$

where, $d_{h x}$ is the distance from the $\mathrm{h}$-bot to the $\mathrm{x}$-bot, measured by the h-bot, and $d_{c} x$ is the distance between the center-bot and the x-bot, which can be calculated by the h-bot after they propagate their relative positions.

By far, three robots in the swarm are aware of their position, which will enable all robots that can communicate with these three robots to calculate their relative positions using trilateration. Once each robot calculates its relative position, it propagates its estimated positions to its neighbours, and eventually the position information is propagated through the swarm. Whenever one of the $\mathrm{x}$-bot or h-bot leaves the neighbourhood of the center-bot, the center-bot will determine a new pair of of $x$-bot and h-bot and propagates their IDs. Then a new procedure for calculating and propagating the positional information will be triggered. In section III-D we explain how the center-bot starts a new procedure for self-localization.

\section{MOTION COORDINATION}

In the previous section, we have proposed a methodology for localization of a swarm of immobile minimalist robots. In the following, we extend the method for localization of mobile robots.

For localization and coordination of minimalist robots, some robots must stay still for a while to serve as beacons for others for the following reasons. First of all, because of limited computational and communicational capacity, it takes time to calculate the position and propagate it to neighboring robots. If all robots are moving, the displacements of the robots during this time will significantly impact the accuracy of the estimated positions using trilateration. Moreover, the lack of the capability to observe other robots velocity and heading leads to increased number of collisions between the robots when all robots are moving. Finally, it is difficult to approach a moving target robot based on the distance measurement when the velocity of the target robot is unknown.

Consequently, there will be a number of immobile robots surrounding each moving robot to avoid collisions between two mobile robots. In this way, the mobile robot will also have sufficient number of immobile neighbours so that it is able to localize itself. This idea can reduce the number of collisions and ensure that all mobile robots receive positional information from temporary immobile neighbours.

Fig. 1 provides an illustrative example, where a moving robot in blue is surrounded by a "polygon" of immobile robots in green inside a region that is within its communication range $R_{c}$. These neighboring robots are termed the first order natural neighbours. To maximize the number of mobile robots, it is desired that only the first order natural neighbours of a mobile robot stay still. Thus, a robot shall be able to distinguish between their first order natural neighbours with other neighboring robots. Therefore, the next step is to identify the first order natural neighbours for each robot. In the following, we implement the Delaunay triangulation method to generate a graph that links each robot to its first order natural neighbours.

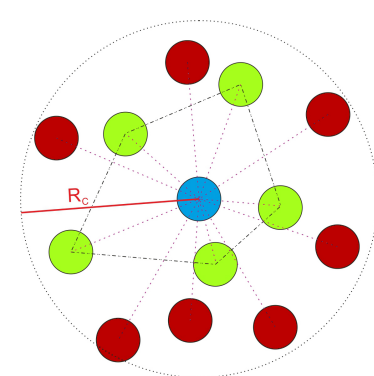

Fig. 1: First order natural neighbours (agents in green) form the operational polygon. 


\section{A. Triangulation}

For a planar straight-line graph $G=(V, E)$, Delaunay triangulation returns a subgraph $G_{D}=\left(V, E^{\prime}\right)$, where $E^{\prime} \subseteq E$ such that each node $v_{i} \subseteq V$ is linked to its first order natural neighbours but not high order neighbours [31]. This information can be useful in many ways including finding a precise edge layer or managing the flow of information through a swarm. Here, we want to utilize triangulation to design a motion schedule that divides the robots into two groups, one mobile and the other immobile, where all mobile robots are surrounded by immobile ones.

Each robot carries out triangulation once each of its neighbours has localized itself and broadcasted a message containing its estimated position. However, it can happen that a robot fails to localize itself because of the lack of three positionaware robots in its neighbourhood or because of missing message in communication. In this case, the robot will wait for a certain period of time before triangulation. Therefore, a robot performs triangulation either when all its neighbours are localized or when a certain period of time is passed. We adopted the Delaunay triangulation algorithm introduced by Lee and Lin [31] in this work.

Algorithm 1 is the pseudo-cod for Delaunay triangulation. Assume $\boldsymbol{T}_{v}$ is initialized using the positional information received from the neighbouring nodes of node $v$. Thus, $\boldsymbol{T}_{v}^{i, p o s}$ stores the $i^{t h}$ neighbours estimated position, $\boldsymbol{P}_{u_{i}}$. In the same way, $\boldsymbol{T}_{v}^{i, i d}$ stores the associated ID of the $i^{t h}$ neighbour, $I D_{u_{i}}$. Let $\boldsymbol{S}_{v} \subset \boldsymbol{T}_{v}$ be the nodes that are linked to node $v$ in the Delaunay graph $G_{D}$. At the begining, the algorithm sort the elements in $\boldsymbol{T}_{v}$ based on increasing angle. It has been proved that the link to the nearest neighbour, $\operatorname{Min}\left(\overline{v u_{i}}\right)$, is a member of $E^{\prime}$. Therefore, the array $\boldsymbol{T}_{v}$ is shiftted till the nearest neighbour locates in the beginning of the array. Then, a local optimization procedure (LOP) is carried on every three radially consecutive neighbouring nodes starting from the nearest neighbour to remove the links that are not in the Delaunay graph from $\boldsymbol{T}_{v}$. We defined a function $\operatorname{LOP}\left(\boldsymbol{P}_{v}, \boldsymbol{P}_{u_{i}}, \boldsymbol{P}_{u_{i+1}}, \boldsymbol{P}_{u_{i+2}}\right)$, where $\boldsymbol{P}_{v}$ is the position of node $v$, and $\boldsymbol{P}_{u_{i}}, \boldsymbol{P}_{u_{i}+1}$ and $\boldsymbol{P}_{u_{i+2}}$ are the positions of three radially consecutive neighbouring nodes of node $v$. LOP returns true if $\boldsymbol{u}_{\boldsymbol{i}+\mathbf{1}}$ is a member of $\boldsymbol{S}_{v}$, and returns false if it is not. Algorithm 1 presents the pseudo code of this procedure.

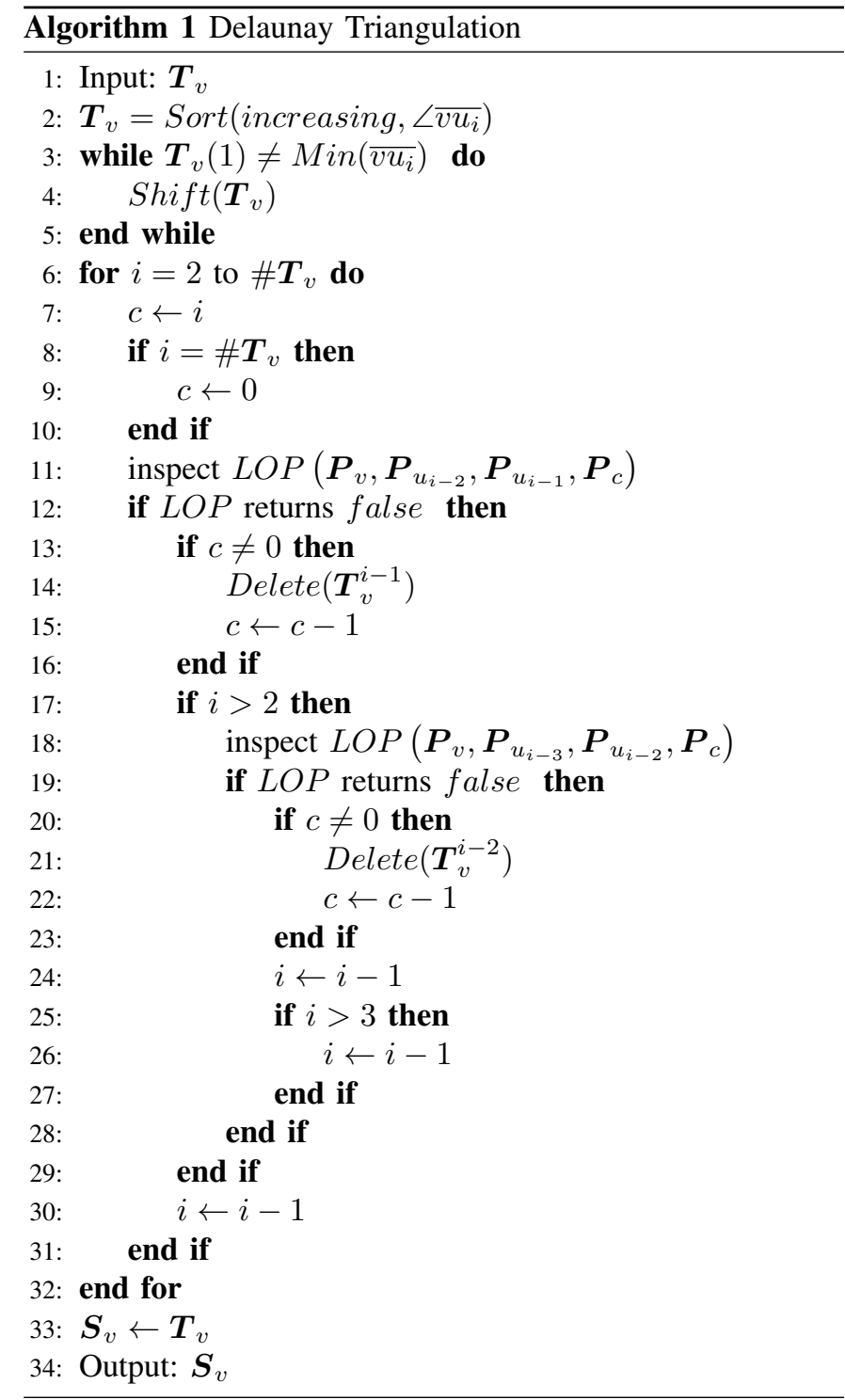

\section{B. Motion Scheduling}

After performing localization and triangulation, a robot is able to distinguish its immediate neighbours. In the following, a motion scheduling is required to make sure that when a robot becomes mobile, its immediate neighbours must remain immobile. The motion scheduling strategy also ensures that an immobile robot will be switched to mobile once all its first order natural neighbours has become mobile. 


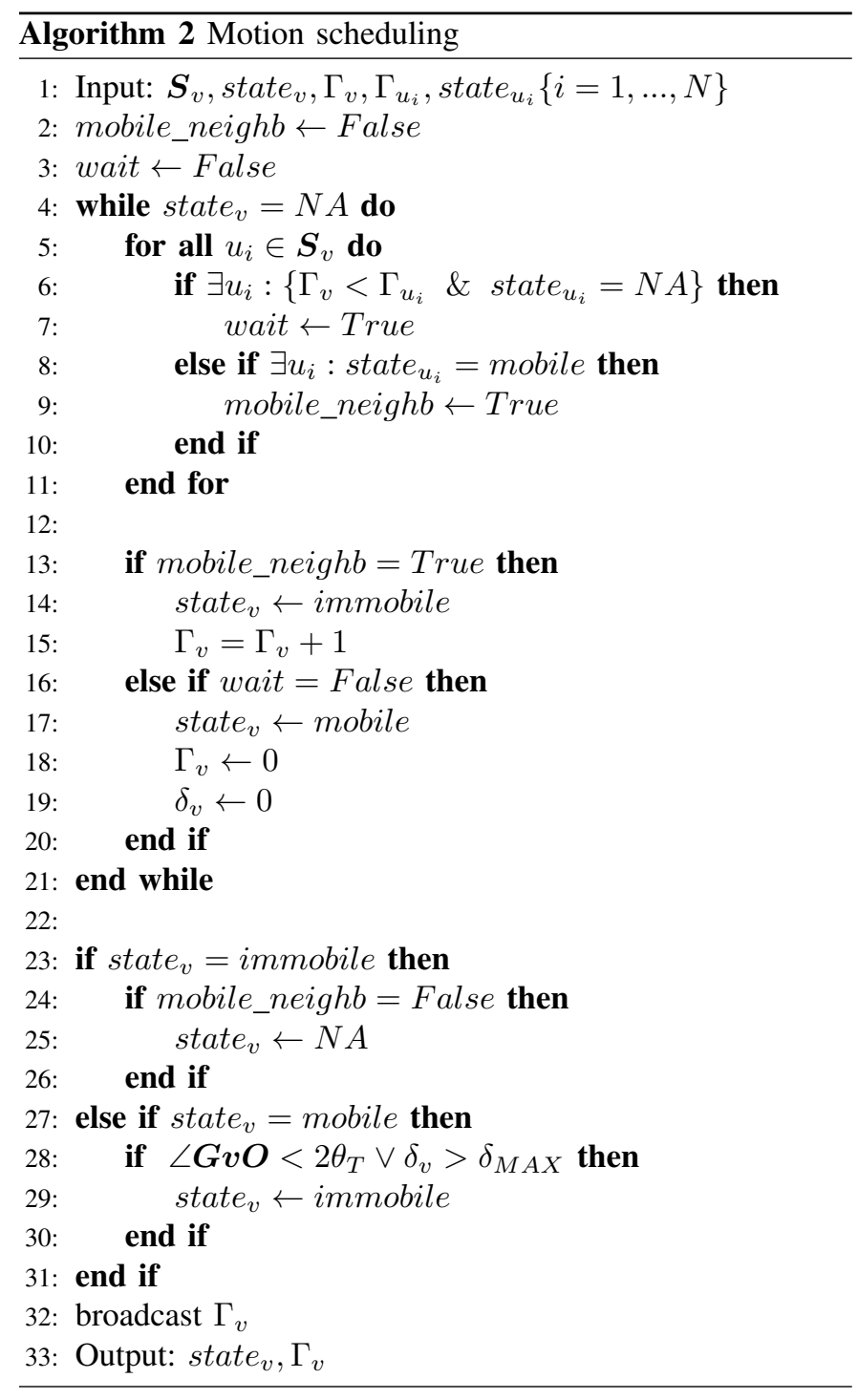

Algorithm 2 provides the pseudo code of motion scheduling. The flag state $v$ indicates the movement status of the robot, which can be mobile, immobile or undetermined. state $_{u_{i}}$ denotes the state of the $i^{t h}$ neighbour in $\boldsymbol{S}_{v}$. In this algorithm, if a robot detects a mobile robot among its first order natural neighbours, it will become immobile. If there is no immobile robot in its neighbouring nodes in the Delaunay graph, a robot checks its neighbour's waiting counter $\Gamma_{u_{i}}$ with it own, $\Gamma_{v}$. This counter is set to zero once a robot becomes mobile, and added by one every time when one of the robots immediate neighbours becomes mobile. The robots communicate this number with each other. In this way, a robot can realize if it has been waiting to become mobile longer than its immediate neighbours. If there is no mobile immediate neighbour, the waiting counter can be used to determine if a particular robot should become mobile.

By contrast, a mobile robot will become immobile, if a certain period of time, indicating by $\delta_{M A X}$, is elapsed or if no progress can be made towards the goal without disconnecting or colliding with its immediate neighbours $\left(\angle \boldsymbol{G} \boldsymbol{v} \boldsymbol{O}<2 \theta_{T}\right)$. $\delta_{v}$ denotes the elapsed time since a robot became mobile. We will explain about $\angle \boldsymbol{G} \boldsymbol{v} \boldsymbol{O}$ and $\theta_{T}$ in the next section.

\section{Motion Planning and Collision Avoidance}

In this session, we aim to design a motion planning method to achieve the following objectives. First, it should be able to preserve the swarm connectivity. Second, it should minimize the number of collisions. Third, it should allow for the maximum progress towards the target during each motion cycle.

To avoid collisions with other mobile robots, each mobile robot must remain inside the operational polygon defined by its immediate immobile robots. To this end, a minimum distance $d_{\min }$ to it immediate neighbours needs to be defined. In addition, we define a maximum distance $d_{\max }$ from a robot's immediate neighbours for preserving swarm connectivity. To maximize the progress towards the target during each motion cycle, each robot will calculate an alternative direction along which the robot can still progress without violating the constraints. In the following, we will discuss this idea in greater detail.

Let $\overrightarrow{\boldsymbol{u}_{i} \boldsymbol{v}}$ be a member of the vector set $\mathcal{O}$ if it satisfies one of the two conditions:

$\mathcal{O}=\left\{\begin{array}{lllll}\text { If } & \left|\overline{\boldsymbol{v} \boldsymbol{u}_{\boldsymbol{i}}}\right|<d_{\min } & \vee \angle \boldsymbol{G} \boldsymbol{v} \boldsymbol{u}_{\boldsymbol{i}}<\frac{\pi}{2} & \text { Then } & \overline{\boldsymbol{v} \boldsymbol{u}_{\boldsymbol{i}}} \in \mathcal{O}, \\ \text { If } & \left|\overline{\boldsymbol{v} \boldsymbol{u}_{\boldsymbol{i}}}\right|>d_{\text {max }} & \vee \angle \boldsymbol{G} \boldsymbol{v} \boldsymbol{u}_{\boldsymbol{i}}>\frac{\pi}{2} & \text { Then } & \overline{\boldsymbol{v} \boldsymbol{u}_{\boldsymbol{i}}} \in \mathcal{O},\end{array}\right.$

Assume $\widehat{G}$ is the unique vector pointing the target direction from the robot, the following equation calculates the alternative direction $\overrightarrow{\mathcal{H}}$ in which the robot can move:

$$
\begin{aligned}
& \overrightarrow{\boldsymbol{O}}=\sum_{\overline{\boldsymbol{v} \boldsymbol{u}_{\boldsymbol{i}} \in \mathcal{O}}} \frac{\overline{\boldsymbol{v} \boldsymbol{u}_{\boldsymbol{i}}}}{\left|\overline{\boldsymbol{v} \boldsymbol{u}_{\boldsymbol{i}}}\right|} \\
& \left\{\begin{array}{l}
\text { If } \angle \boldsymbol{G} \boldsymbol{v} \boldsymbol{O}>=2 \theta_{T} \quad \text { Then } \overrightarrow{\mathcal{H}}=\widehat{\boldsymbol{G}}-\frac{\overrightarrow{\boldsymbol{O}}}{|\overrightarrow{\boldsymbol{O}}|} \\
\text { If } \angle \boldsymbol{G} \boldsymbol{v} \boldsymbol{O}<2 \theta_{T} \quad \text { Then } \text { state }_{v} \leftarrow i m \text { mobile }
\end{array}\right.
\end{aligned}
$$

In the above equation, $\theta_{T}$ specifies the maximum difference between the alternative direction and the goal direction that is allowed, $\mathcal{O}$ is a set containing all directions that violate the constraints, and $\vec{O}$ is the resultant directions. Fig. 2 illustrates the direction of the vectors engaged in motion planning. To estimate the goal direction, $\hat{G}$, a robot uses information from their immediate neighbours only. This information can be either some environmental variables such as intensity of a chemical, heat and light, or some internal variables that are calculated using neighbours neighbours information. An example of such internal variable is the artificial morphogen that we will explain in more detail in section $\mathrm{V}$.

With the aim of defining an additional term for Eq. (5) that provides more control over preserving connectivity, we define vector $\overrightarrow{\boldsymbol{F}}$, termed coherence force, as follows:

$$
\overrightarrow{\boldsymbol{F}}=\frac{\sum_{u_{i} \in \boldsymbol{S}_{v}} \overrightarrow{v u_{i}}}{\sum_{u_{i} \in \boldsymbol{S}_{v}}\left|\overrightarrow{v u_{i}}\right|} .
$$

Taking the coherence force into account, the alternative direction $\overrightarrow{\mathcal{H}}$ is updated by following equation:

$$
\overrightarrow{\mathcal{H}^{\prime}}=\overrightarrow{\mathcal{H}}+\beta \overrightarrow{\boldsymbol{F}}
$$

As the coherence force is divided by the sum of the immediate neighbours distances, its magnitude depends on 


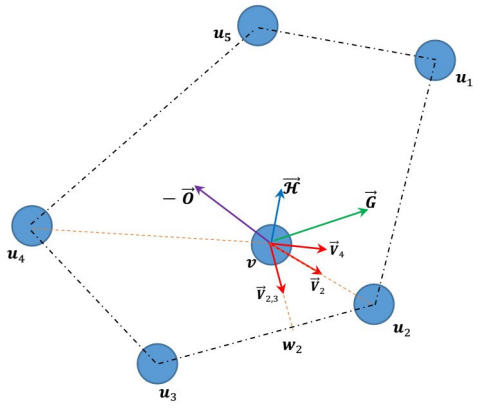

Fig. 2: Vectors engages in motion planning of robot $v$. The dash-dot lines represent the polygon defined by the immediate neighbours of robot $v$.

the number of the immediate neighbours and their distances. Hence, this force becomes larger at the boundary of the swarm where there are fewer immediate neighbours, or in an area in which there are a large number of robots resulting in a much shorter distance to their immediate neighbours. Fig. 3 shows an illustrative example of the magnitude and direction of the coherence force for a swarm with an irregular shape in the simulations. From the figure, we can see that the direction of the coherence force points towards the center of the swarm when the robot is at the boundary and to some vacant space when it is inside the polygon.

A positive value for $\beta$ can result in a tendency of moving towards the center of the polygon defined by the immediate neighbours. For edge robots, which are outside of this polygon, the coherence force is towards the center of the swarm, which can promote connectivity. In section IV, we will investigate how the value of $\beta$ influences the shape of the swarm during flocking. It is found that negative $\beta$ values will prevent the swarm from becoming elongated.

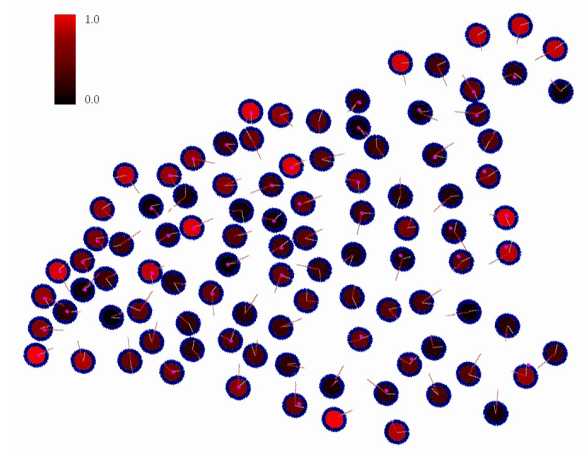

Fig. 3: The magnitude and direction of the coherence force. The red lines indicate the direction of the coherence force.

\section{Communication of Information}

Table I provides the structure of the message adopted for communication of information between robots. A robot sets the first byte of its message always equal to its ID. In this way, the robots can identify their neighbours and are able to recognize from which neighbour a message is coming. A
TABLE I: Structure of Messages

\begin{tabular}{|c|c|c|}
\hline byte 1 : & & \\
\hline byte 2 : & & yte \\
\hline byte 3 : & Free bits & Message type \\
\hline byte 4 : & float & $\mathrm{N}$ byte \\
\hline byte 5 : & float & byte \\
\hline byte 6 : & float & $N$ byte \\
\hline byte 7: & float & byte \\
\hline byte 8 : & & \\
\hline byte 9 : & & \\
\hline
\end{tabular}

robot uses its second byte to broadcast its current internal state to its neighbours. Each bit of the second byte is used as a flag to indicate a certain on-going action or a particular condition is met. For example, a number of bits are allocated to indicate whether a robot is mobile or immobile, localized or not, or whether a robot has detected right-handed or lefthanded coordinate system. The first four bits of the third byte are used for determination of the types of messages. From the message type, a robot can determine to which variable each byte is related, and also their range of change. To communicate the float number, we reduce the precision of floats into 15 binary digits, and reserve one digit for carrying the sign. In this way, the smallest reportable change of a variable is equal to the changing range of the variables divided by $2^{15}$.

As noise produced by inaccurate distance measurements and missing messages accumulates, there is a need for recalculation of the positional information from time to time. For this purpose, a wave of new positional information starts to propagate from the centre core to other robots. Whenever position information needs to be recalculated, a new coordinate system may be set up and therefore, the positional information previously stored will become invalid. Thus, a signalling message is triggered by the center robot and spreads throughout the swarm to perform triangulation and localization. To this end, we have introduced a specific message type so that whenever a robot receives a this type of message, it will first broadcast the same type of message and ignores messages of this type received within a certain period of time. In this way, this message is received and sent out by each robot only once.

\section{SIMULATION RESULTS}

To evaluate the performance of the proposed algorithm, a simulation environment was developed using $\mathrm{C} / \mathrm{C}++$ programming languages in the Visual Studio environment with a 2D graphical user interface in standard development library (SDL). One important advantage of the simulator is that the code implemented in the simulator can directly be transplanted to the real Kilobots without any change.

We have performed a large number of simulations to tune the parameters of the algorithm. Table II lists the parameter values found to produce good performance.

It should be noted that we did not consider taking any additional measures to recover the lost robots. Once a robot cannot see any localized robot in its neighbourhood, it simply stops moving. We could add some random walk behaviours, however, this work is more concerned with how efficient the proposed algorithm is in maintaining the swarm connectivity independent of other strategies. 
TABLE II: Parameter values used in the simulations

\begin{tabular}{lll}
\hline \hline Parameter & Description & $\begin{array}{l}\text { Chosen } \\
\text { value }\end{array}$ \\
\hline$d_{\min }$ & Eq. (3) & $47 \mathrm{~mm}$ \\
$d_{\max }$ & Eq. (3) & $80 \mathrm{~mm}$ \\
$\theta_{T}$ & Eq. (5) & $\pi / 5$ \\
$\beta$ & Coherence force coefficient Eq. (7) & -0.2 \\
$\delta_{M A X}$ & Maximum allowed time to be contin- & $30 \mathrm{~s}$ \\
\hline
\end{tabular}

\section{A. Movement Efficiency}

To examine movement efficiency of the swarm, we count the number of times each robot becomes mobile during 1000 motion steps. Fig. 4 shows the percentage of the robots becoming mobile for different swarm sizes. From the figure, we can see that for smaller swarms, the chance of each robot becoming mobile is higher than bigger swarms. Note, however, that the mobilization percentage converges to 20 as the size of the swarm increases. In other words, approximately $20 \%$ of the robots are mobile at each time step.

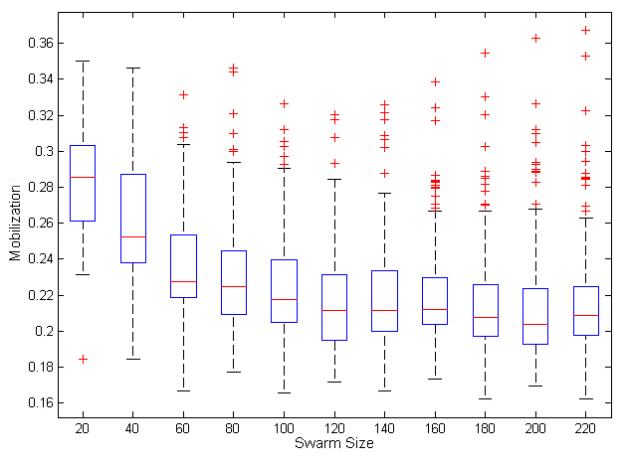

Fig. 4: Motion efficiency for different swarm sizes.

\section{B. Movement Consistency}

We evaluate the movement consistency of the proposed algorithm using a scenario in which the robots are flocking towards a light source. In this scenario, each robot in the simulation is equipped with an omnidirectional light sensor, and a light source is placed at a certain position in the considered environment. With the omnidirectional light sensors, the robots are able to detect the direction of the light source provided that different light intensities can be sensed at different positions. In reality, the change in light intensity in different places that are not very distant from each other is minor due to noise in measurements. Therefore, measurements from other robots are required to achieve a reliable estimation of the light source direction. For this reason, during each motion step, all immobile robots send messages containing their measurements of the light intensity. The light source direction, $\overrightarrow{\boldsymbol{L}}$ can be estimated as follows:

$$
\forall u_{i} \in \boldsymbol{T}_{v}: \overrightarrow{\boldsymbol{L}}=\sum\left(I_{u_{i}}-I_{v}\right) \frac{\left(\boldsymbol{P}_{u_{i}}-\left(\boldsymbol{P}_{v}\right)\right.}{\mid \boldsymbol{P}_{u_{i}}-\left(\boldsymbol{P}_{v} \mid\right.},
$$

where, $I_{v}$ is the light intensity measured by the center robot, and $I_{u_{i}}$ is the light intensity measured by robot $u_{i}$.

\section{Effect of the Coherence Force}

As previously discussed, the presence of the coherence force is essential for prevent the swarm from becoming elongated. In Fig. 5 the shapes of the swarm are presented for different values of $\beta$ after one meter of the mass center displacement. From these results, we can see that a larger $\beta$ increases the lateral force towards the mass center, and as a result, the swarm becomes elongated. By contrast, a negative value for $\beta$ changes the direction of the outset robots slightly outwards, which enables the edge robots to use free space outside the swarm, resulting in a more round-shaped swarm. In the rest of our experiments, we set $\beta$ to -2 .

\section{Connectivity Metric}

For minimalist robots, it is important that they stay communicationally connected as aggregation is difficult once a robot is disconnected from the swarm in the absence of global positional information. To quantitatively assess the performance of the proposed algorithm in maintaining the swarm, here, we propose a metric that is sensitive to every single loss of communication. To the best of our knowledge, no such metrics have been reported in the swarm robotics literature. The new metric for measuring connectivity of a swarm with a limited communication range defined as follow:

$$
\text { connectivity }=\frac{\left|E_{c r}\right|}{\left|E_{\infty}\right|},
$$

where $E_{c r}$ represents the number of links in the Delaunay graph when the communication range is equal to $c r$, while $E_{\infty}$ is the number of links in the Delaunay graph when the communication range is equal to infinity. Fig. 6 plots the value of the proposed connectivity metric for different swarm arrangements. We can see that this metric is fairly sensitive to the loss of communication between robots as well as the swarm density. The connectivity metric becomes larger when the robots move closer to each other.

In another set of experiments, we disconnect robots one by one from a swarm of 100 robots and measure the connectivity. The result shows that our metric is linearly bounded with the number of lost robots.

\section{E. Effect of noise}

The swarm robotic system in this work is subject to four major sources of noise, including noise in distance measurements, inaccurate feedback-less motion, missing messages from neighbours, and noisy light sensing. Missing messages usually occur when two robots send messages at the same time. Therefore, more messages will be missing as the number of neighboring robots increases. The chance of missing a message to be equal to $5 \log _{10} N$, where $N$ is number of neighbours. Moreover, because of memory limitation in Kilobots, it is impossible to save the information received from all neighbours. Hence, the maximum number of neighbours data buffer is limited to 16 which is another source of missing messages. If the number of neighboring exceeds 16 , each robot throws away the data received from the farthest neighbour so 

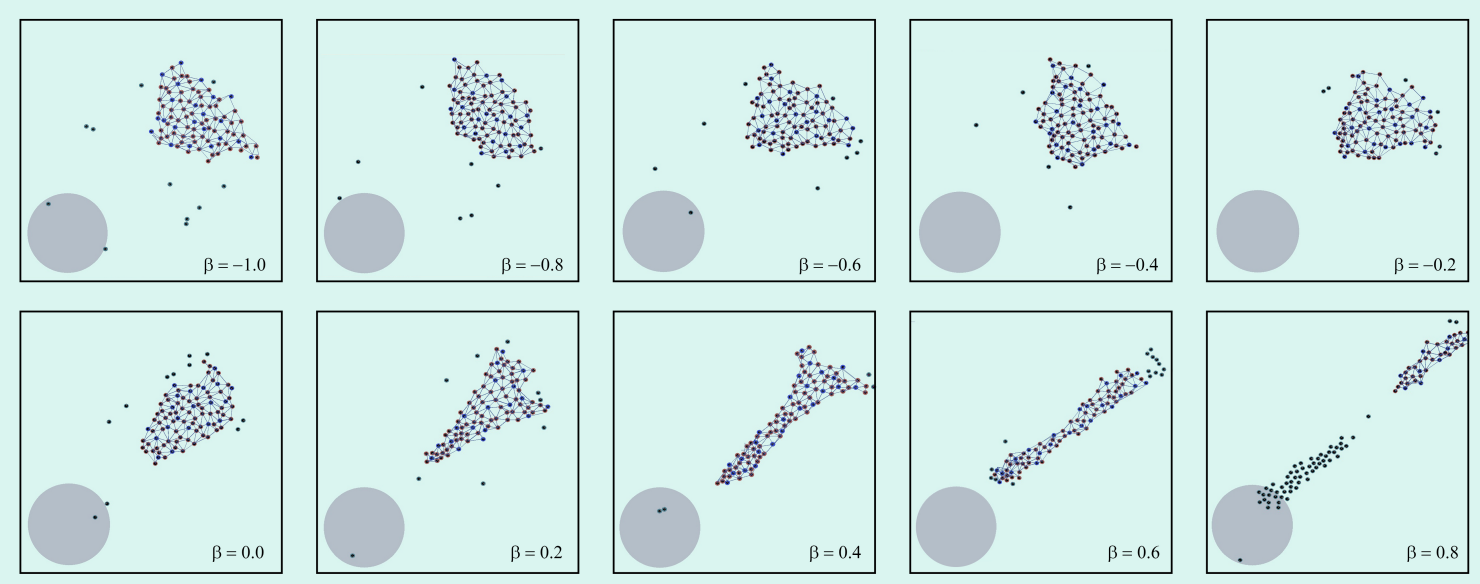

Fig. 5: The shapes of the swarm after one meter of the mass centre displacement for different values of $\beta$. The grey circle indicates the initial position of the swarm.
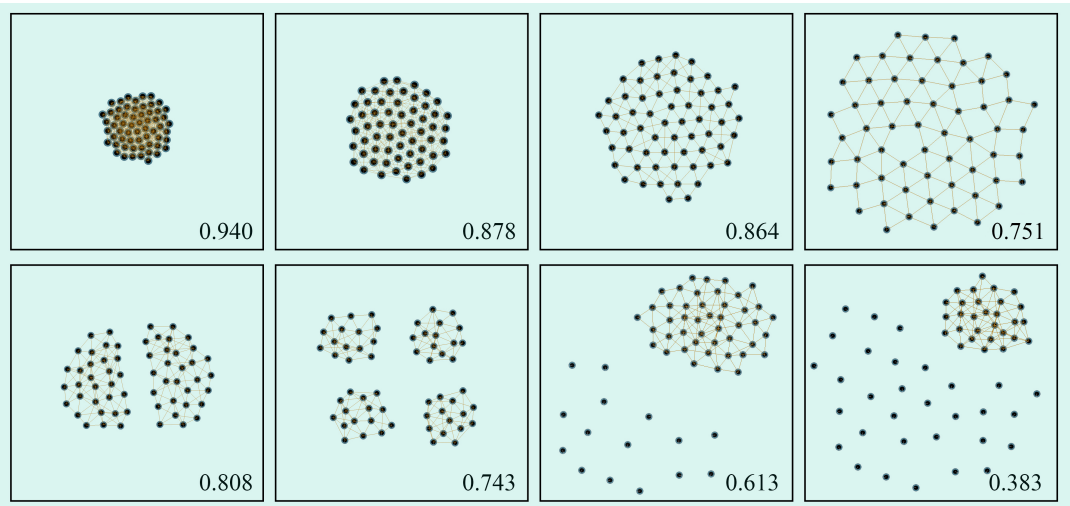

Fig. 6: Connectivity metric for different swarm arrangements. The robots within their communication range are connected by a line.

as to keep the data from the nearer neighbors. Once more, because of memory limitation, it is costly to save measured distance in float numbers. Therefore, the distance to each neighbour is saved as a 8-bit integer number in millimetre. Thus, the distance measurement has a systematic error of $\pm 0.5 \mathrm{~mm}$.

In the following, we study the effect of increasing measurement noise on the connectivity and flocking speed of a simulated swarm, where motion noise and light sensing noise are neglected. We consider swarms consisting of 60 simulated Kilobots, which are initially placed within a circle large enough to allow robots to have at least four centimeter distance from the body center of each other. We let each simulated swarm flock towards the light source till its center of mass displaces one and half meters, and then calculate the connectivity. Each experiment is repeated for 40 times, and the mean and standard deviation of the connectivity and displacement over the 40 runs are given in Fig 7(a) and Fig 7(b), respectively.

The results show that the distance measurement noise up to $\pm 1 \mathrm{~mm}$ does not significantly affect the performance. However, when the distance measurement noise is larger than $\pm 2 m m$, the performance seriously degrades. By adding
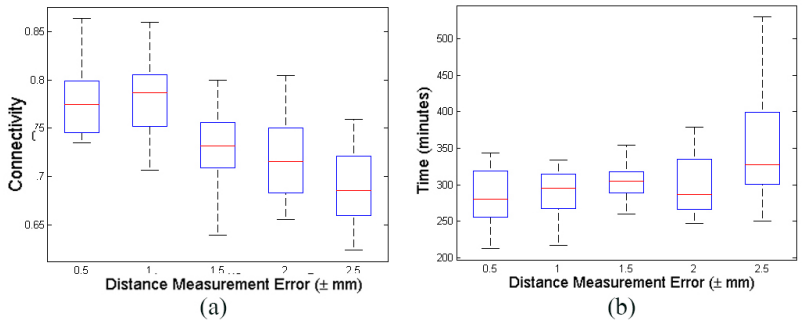

Fig. 7: Effect of distance measurement noise on a) connectivity preservation, and a) flocking speed.

more noise to the distance measurements, the error in selflocalization increases. Erroneous positional information leads to inaccuracy in heading and goal direction estimation, resulting in more wrong motion decisions. In extreme cases, the graph formation can also be affected, where the agents build graph links with non-immediate neighbours. Erroneous selflocalization also increases collisions, which decreases connectivity and slows down the movement. It should be noticed that, if a robot does not have enough localized neighbours for selflocalization, it will become immobile.

Motion without feedback is one important limitation of 
minimalist robots like Kilobot. Implementation of motion encoders is costly in terms of price and size. Without internal motion feedback, it is not straightforward to estimate velocity. Moreover, the velocity of each individual robot also heavily depends on many other factors including the characteristic of the surface, battery level, collision with other robots. Regarding the noisy distance measurement and non-directional sensing, the algorithms for velocity calculation are inaccurate and also computationally intensive. Therefore, algorithms for self-organizing minimalist robots must be tolerant of noisy motion. In the previous simulations, it was assumed that the robots know their velocity precisely. Here, we limit the amount of distance measurement to $\pm 1.0 \mathrm{~mm}$ and gradually increase the inaccuracy of the velocity, which is realized by adding a random noise to the real velocity of a robot. In the simulations, we limit the upper bound of this random number to a certain percentage of the approximated velocity. We can increase this upper bound in order to increase the noise level.

Fig. 8 (a) and 8 (b) show the effect of increasing motion noise on the flocking performance. From these results, we can see that the connectivity and displacement time are not considerably affected when the motion noise is below 20 percent. For even a higher level of motion noise, the proposed algorithm is still able to maintain the swarm connectivity to a certain degree. However, the elapsed time for completing the displacement increases significantly. The insensitivity to motion noise in maintaining the swarm connectivity may be attributed to the factor that each mobile robot is able to update its positional information and estimate the motion speed with the help of its immobile neighbors. However, by adding more and more noise, it becomes more likely to make mistakes in estimating the handedness of the coordinate system.

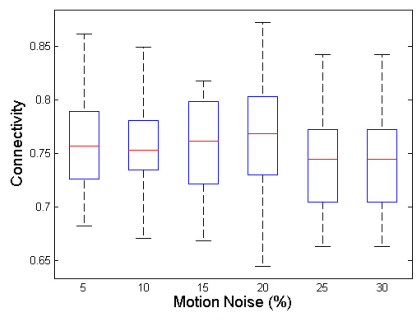

(a)

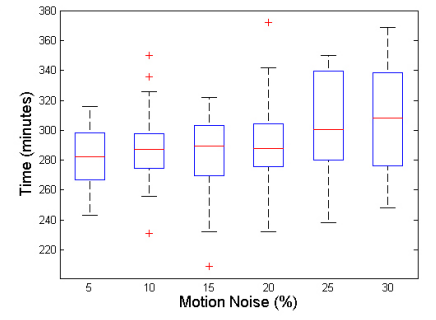

(b)
Fig. 8: Effect of motion noise on (a) connectivity maintenance and (b) flocking speed.

A mistake in estimation of handedness will result in a wrong motion decision. As the number of the wrong motion decisions increases, the time needed for aligning with the goal direction and overall swarm displacement will also increase. Similarly, mistakes in estimation of handedness will also increases the chance of collision, which itself deteriorates the accuracy of the expected position. Hence, in this algorithm, the noise in motion becomes substantially harmful when it starts deteriorating the ability of the proposed algorithm to detect the correct handedness of the local coordinate system.

Finally, we consider noise in sensing the ambient light intensity. We set the noise level in distance measurement and the motion estimation to $\pm 1 \mathrm{~mm}$ and $\pm 15 \%$, respectively, and gradually increase the level of the noise in sensing light intensity to examine the influence of the noise on the connectivity and the flocking speed. As can be seen in Fig. 9(a) and 9(b), the level of light sensing noise has a severe effect on both the connectivity and flocking speed. The advantage of collective determination of the light direction is that robots do not need to move around to estimate the gradient direction. But they can communicate their measured intensity of light with each other to figure out the increasing direction of light intensity. However, as light and chemical gradients in reality are usually very smooth, and also because of the limited communication range, this estimated direction is still vulnerable to noise. In our simulation the density of light increases by 12 percent between two points aligned in increasing direction of light with a distance equal to the maximum communication range. When the amount of noise is comparable with this slope, the percentage of noise in the sensing of ambient light intensity strongly influences the accuracy of the estimated light direction. We increase the light noise gradually to 20 percent of its actual value. Since the light sensing noise seriously affects the flocking performance, this error increases the number of motions that do not effectively bring the robot closer to the light source. Consequently, the flocking speed toward the light source decreases. In particular, at the boarder of the swarm, where the edge robots have a fragile connection with a smaller number of neighbours, the inaccuracy in estimating the target direction can lead to disconnection of the edge robots from the swarm. As a result, the connectivity of the swarm directly decreases when the noise level in light sensing increases.

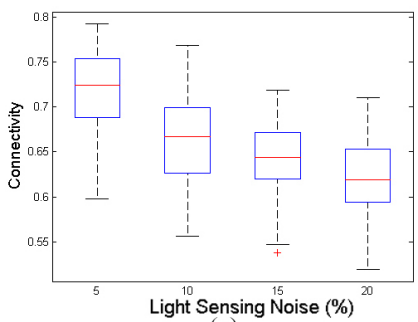

(a)

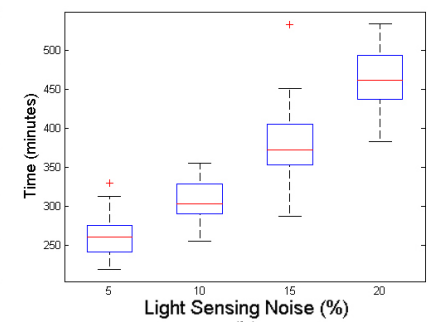

(b)
Fig. 9: Effect of light sensing noise on (a) connectivity preservation, and (b) flocking speed.

\section{F. Scalability}

We investigate the scalability of proposed algorithm to the number of robots in the swarm. That is, we evaluate the change in flocking performance as the number of robots in the swarms. We consider different swarm sizes ranging from 30 to 200 robots. The levels of noise in distance measurement, motion estimation, and light sensing are set to $\pm 1.0 \mathrm{~mm}, \pm 20 \%$ and $\pm 5 \%$, respectively.

The results on flocking performance are presented in Fig. 10. It can be observed that the connectivity of the swarm increases as the size of the swarm increases up to 150 robots. When the size is larger than 150, the swarm connectivity reduces. This can be attributed to the fact that the connectivity of small swarms is very sensitive to missing robots. Hence, for small swarms, the connectivity improves as the number of 
robots increases. However, because the positional information spreads from the center robot, the error in localization accumulates for robots farther away from the center. Typically, self-localization of edge robots is less accurate than the inner robots. As the size of the swarm increases, the positional information of the edge robots becomes less and less accurate. Thus, as the size of the swarm increases, the edge robots become more likely to be disconnected from the swarm.

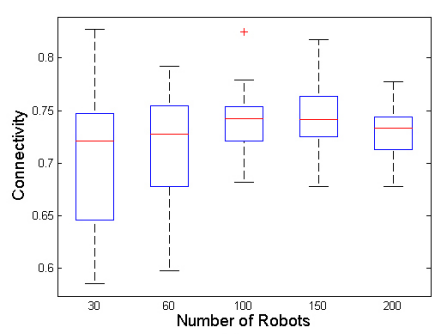

(a)

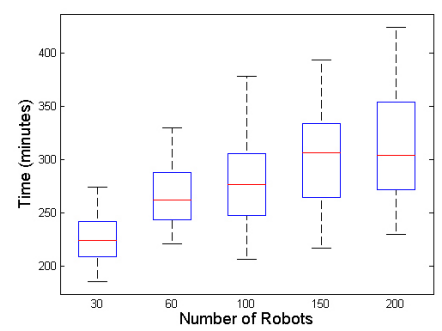

(b)
Fig. 10: Scalability of connectivity preservation (a), and flocking speed (b)

\section{ExPERIMENTAL Results USING Kilobots}

In this section, we evaluate the proposed algorithm on physical Kilobots. Although Kilobots are not applicable to any realworld scenarios, they are one of a few cheap minimalist robots available for proof-of-concept experiments. In the experiment with real Kilobots, the swarm is required to follow a moving target instead of following the light source. This is due to the fact that decreasing the ambient light will dramatically shorten the Kilobots communication range. The reason is that stronger signal amplification is needed in the presence of intense ambient light so that robots can detect signals farther away. Therefore, once a gradient of ambient light is generated on the robots, those at the dark side easily get lost because of a shortened communication range. One additional problem is that the ambient light sensor on the board is located at the backside of the Kilobot. Hence, when a Kilobot is heading towards the light source, it receives less light than when it is moving away from light. Moreover, the light from other Kilobots LED add lots of noise to light sensing in the dark area.

For the above-mentioned reasons, we make use of a gradient of artificial morphogen instead of a gradient of light [30]. Therefore, we place a target robot in front of the swarm as the source of the morphogen. The target moves forward once its number of neighbour is higher than a certain threshold.

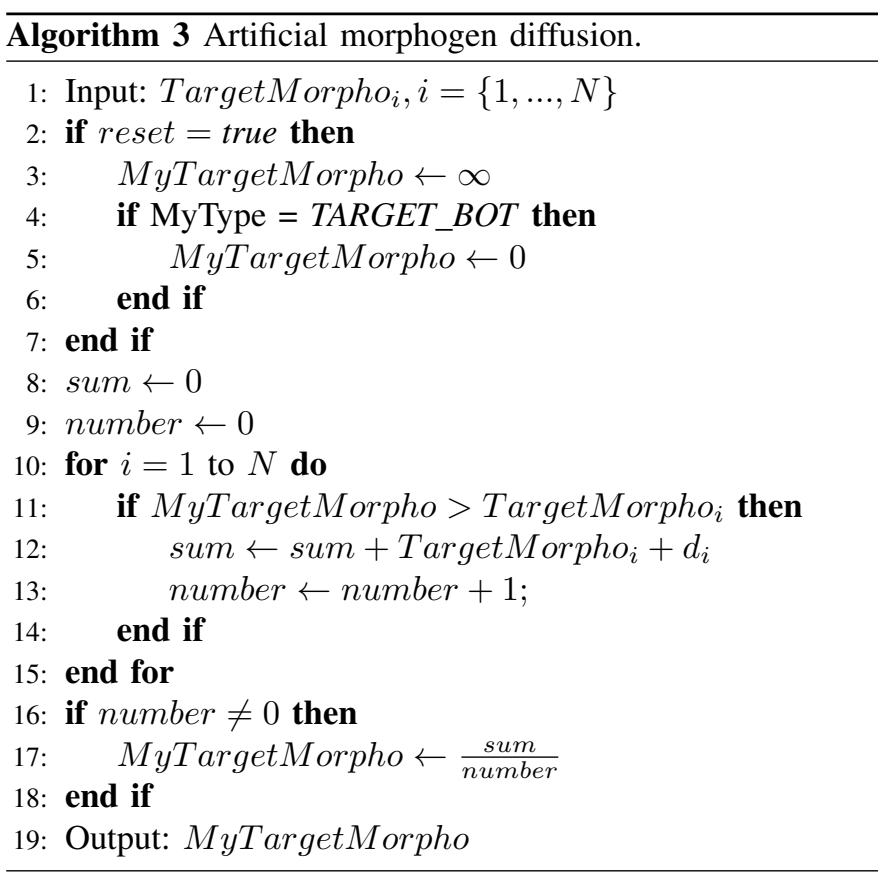

Algorithm 3 describes the process of morphogen diffusion. MyTargetMorpho represents the robot's morphogen concentration, and TargetMorpho $o_{i}$ indicates its $i^{\text {th }}$ neighbour's morphogen concentration. In the beginning, the concentration is set to infinity in all robots except for the target robot, whose concentration is always set to zero. To calculate the concentration, a robot only takes its neighbours with less concentration into account. Through diffusion a gradient of the morphogen is generated across the entire swarm. Fig. 11 illustrates this gradient, which is formed by 60 simulated Kilobots. We consider a noise level of $\pm 2 \mathrm{~mm}$ in distance measurement. Even in this case, the morphogen gradient is quite large and relatively insensitive to the noise. Therefore, we use the morphogen concentration instead of the light intensity and the swarm is required to move toward the target robot.

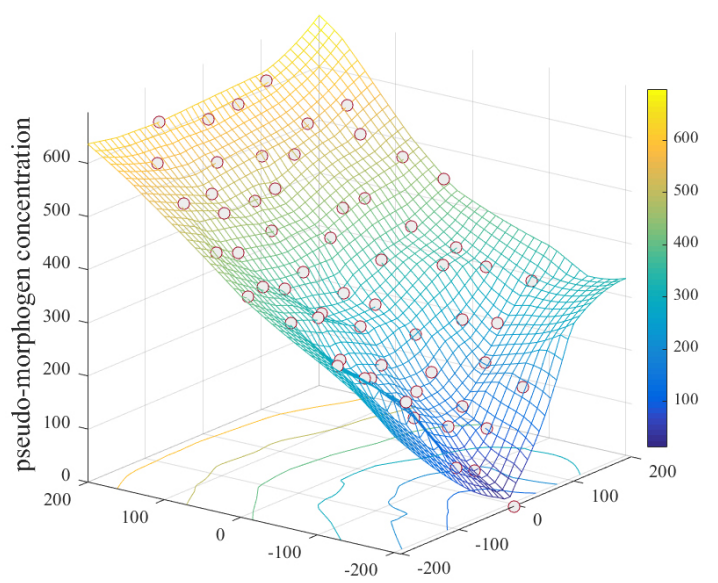

Fig. 11: The concentration of morphogen across a swarm of simulated Kilobots with $\pm 2 \mathrm{~mm}$ distance measurement noise

To evaluate the efficiency of our algorithm, we compare its performance with a swarm of individual robots without 

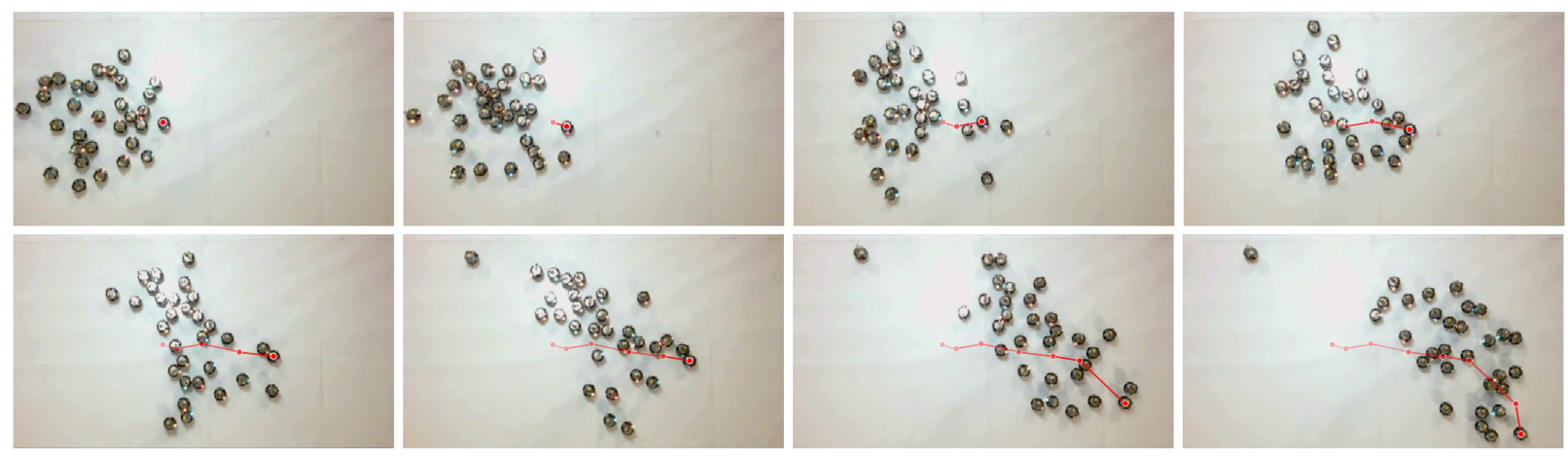

Fig. 12: Implementation of the algorithm for self-organizing coordinated motion scheme for target following using morphogen diffusion. Time increases from top to bottom, and left to right. The red dots in each snapshot represent the location of the target robot in the current and previous snapshots.
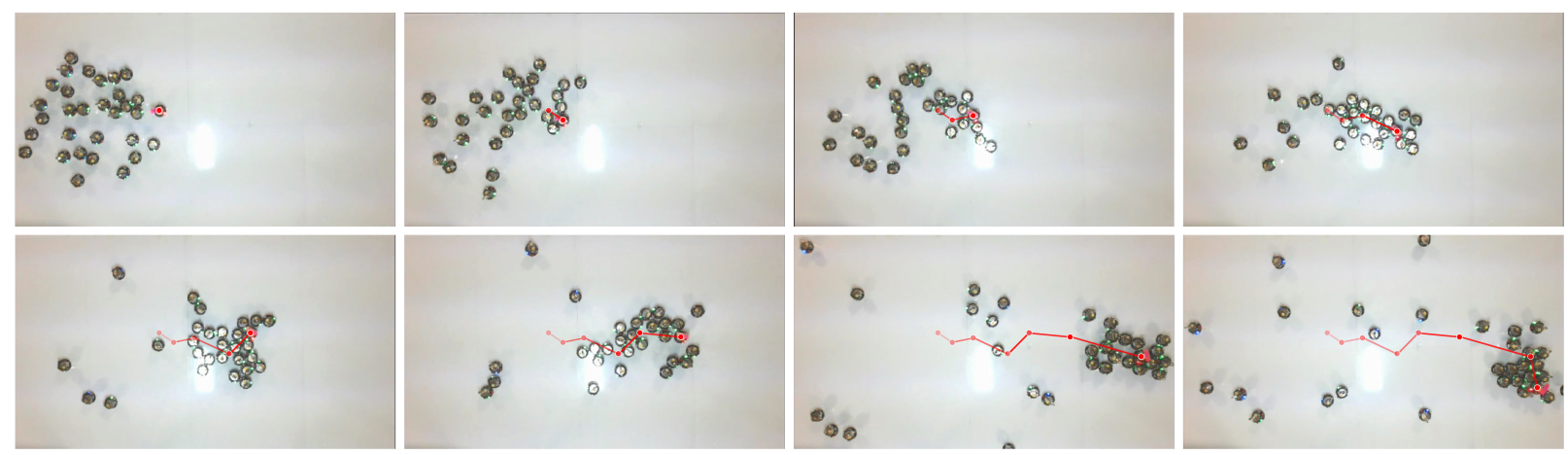

Fig. 13: Implementation of the algorithm for target following using morphogen diffusion without localization and motion scheduling. Time increases from top to bottom, and left to right. The red dots in each snapshot represent the location of the target robot in the current and previous snapshots.

collaborative positional information and motion scheduling. In this scenario, morphogen diffusion is the same as before. Here, the robots try to move in the direction that maximizes the drop in the target morphogen concentration. For maintaining connectivity, a robot makes a turn if the number of neighbors it has decreases below a certain threshold. This is a widely used strategy for connectivity maintenance [32]. As mentioned before, a robot updates its morphogen concentration using information only from the robots with a lower morphogen concentration. Consequently, the robots morphogen concentration stops changing if no robots has a lower level of concentration. In this algorithm, if there is no change in the morphogen concentration after a motion step, a robot will move randomly. Hence, if a robot is disconnected from the swarm, it starts to do a random walk till it finds a robot with a lower target morphogen concentration.

The snapshots of the experimental results for following two targets are shown in Fig. 12 and Fig. $13^{1}$.. From these results, we can see that the robots are able to maintain connectivity even if no motion strategy is specified for missing robots. There are still collisions between robots as expected, because of the short communication range. However, the

\footnotetext{
${ }^{1}$ The movie can be downloaded from the link bellow: https://www.dropbox.com/sh/54nap1t0u7c2b0p/AADKiQzOD8uqkGfx6iSUUK4a?dl=0
}

proposed motion scheduling strategy successfully reduced collision jams, which are really detrimental to connectivity maintenance and flocking performance. Moreover, because of the outward coherence force, the edge robots tend to move ahead of their front neighbours by moving in the direction perpendicular to the target direction. This force prevents the swarm from becoming elongated, resulting in a better flocking and more space for the inner robot of the swarm to move.

In the absence of localization and motion scheduling, collision jams form very quickly after the initiation of target following. The robots in the collision jams lag behind the others, leading to disconnection. Once a group of robots are disconnected from the rest, the robots with the highest target morphogen concentration will move randomly and the others will follow it. This is because the target morphogen concentration does not get updated if there is no robot with a higher level of concentration, as we mentioned before. As can be observed in Fig. 13, there are many robots disconnected from the swarm during target following. Apart from collisions, slow communication and computation power also cause wrong motion decisions and disconnections. This is because if calculation of morphogen concentration takes too much time, the morphogen concentration has changed. In these cases, it is hard to distinguish the direction of the morphogen concentration.

Finally, we calculate the connectivity of the swarm using 


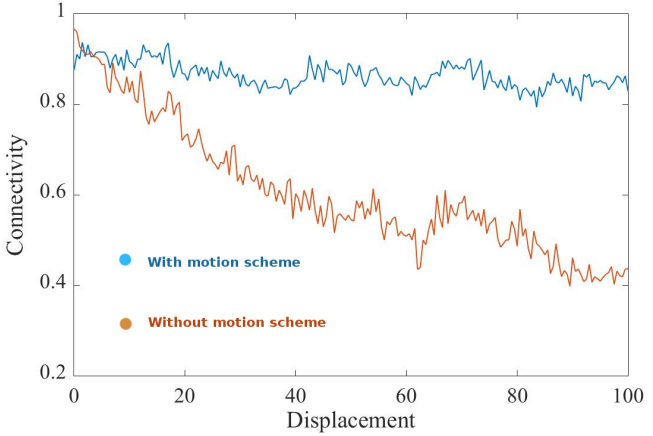

Fig. 14: Comparison of the connectivity of two swarms with and without motion scheme versus the displacement of the target.

the proposed metric, starting from the beginning of target following till the target displaces one meter. The results are depicted in Fig. 14. The experimental results confirm that the proposed algorithm for self-organizing coordinated motion performs much better than the algorithm without using motion scheme. From the figure, we can see that the algorithm without motion scheduling loses it connectivity continuously during the flocking. By contrast, the proposed algorithm is able to maintain the connectivity of the swarm within a certain range.

It is worth noting that the velocity of the target robot is slower in the beginning. This is due to the fact that the robots are initialized in random directions, it takes time before they become approximately aligned with the direction of the target morphogen gradients. But once their heading becomes more and less towards the target and the direction of flocking, the robots can follow the target much faster. In particular, the robot closer to the target usually follow the target faster than others. This can be attributed to the fact that the closer a robot is to the target, the less big the accumulated noise will be in calculating the target morphogen concentration. Thus, there will be fewer wrong motion decisions, and consequently the swarm can follow faster and fewer robots will be disconnected from the swarm. However, the velocity of the target robot needs to be tuned according to the velocity of the back site robots if the gradient is small and noisy. What happens as a result is that the robots in the front of the swarm move close to target and even start to push it forward. Therefore, the velocity of the target robot starts to increase.

\section{CONCLUSION}

We proposed a self-organizing algorithm for coordinated motion of a swarm of minimalist robots that do not have selflocalization and orientation sensing. The algorithm is able to estimate the relative positional and directional information, which is then used to form a polygon of immobile robots inside which a robot can move without collision. In addition, we propose a motion planning strategy, where a coherence force is embedded to maintain a round-shaped swarm during flocking. With the help of the graph theory, we introduced a new metric for measuring the connectivity of robotic swarms. We showed that this metric is able to account for disconnected robots and provides a sensible quantitative measure for swarm connectivity. We analysed the influence of different sources of noise on the swarm connectivity and flocking speed in simulations. Finally, a swarm of physical Kilobots is to used to assess the performance of the proposed algorithm. Artificial morphogen diffusion was implemented to enable the robots to estimate the target direction. We compared the proposed algorithm with the one without localization and motion planing with respect to connectivity maintenance and smoothness of flocking. Our experimental results demonstrate the effectiveness of the proposed algorithm for swarm target following.

\section{REFERENCES}

[1] L. Bayindir, "A review of swarm robotics tasks," Neurocomputing, vol. 172, pp. 292-321, 2016.

[2] H. Oh, A. Ramezan Shirazi, C. Sun, and Y. Jin, "Bio-inspired selforganising multi-robot pattern formation: A review," Robotics and Autonomous Systems, vol. 91, pp. 83-100, 2017.

[3] M. Brambilla, E. Ferrante, M. Birattari, and M. Dorigo, "Swarm robotics: a review from the swarm engineering perspective," Swarm Intelligence, vol. 7, no. 1, pp. 1-41, 2013.

[4] Z. Chen and T. Chu, "Multi-agent system model with mixed coupling topologies for pattern formation and formation splitting," Mathematical and Computer Modelling of Dynamical Systems, vol. 19, no. 4, pp. 388400, 2013.

[5] T. Yasuda, A. Adachi, and K. Ohkura, "Self-organized flocking of a mobile robot swarm by topological distance-based interactions," in System Integration (SII), 2014 IEEE/SICE International Symposium on. IEEE, 2014, pp. 106-111.

[6] E. Ferrante, A. E. Turgut, A. Stranieri, C. Pinciroli, M. Birattari, and M. Dorigo, "A self-adaptive communication strategy for flocking in stationary and non-stationary environments," Natural Computing, vol. 13, no. 2, pp. 225-245, 2014.

[7] Y. Jin, H. Guo, and Y. Meng, "A hierarchical gene regulatory network for adaptive multi-robot pattern formation," IEEE Transactions on Systems, Man, and Cybernetics, Part B: Cybernetics, vol. 42, no. 3, pp. 805-817, 2012.

[8] H. Guo, Y. Jin, and Y. Meng, "A morphogenetic framework for selforganized multi-robot pattern formation and boundary coverage," $A C M$ Transactions on Autonomous and Adaptive Systems, vol. 7, no. 1, p. Article No. 15, 2012.

[9] N. R. Hoff III, A. Sagoff, R. J. Wood, and R. Nagpal, "Two foraging algorithms for robot swarms using only local communication," in Robotics and Biomimetics (ROBIO), 2010 IEEE International Conference on. IEEE, 2010, pp. 123-130.

[10] C. Moeslinger, T. Schmickl, and K. Crailsheim, "A minimalist flocking algorithm for swarm robots," in Advances in Artificial Life. Darwin Meets von Neumann. Springer Berlin Heidelberg, 2009, pp. 375-382.

[11] A. E. Turgut, H. Çelikkanat, F. Gökçe, and E. Şahin, "Self-organized flocking in mobile robot swarms," Swarm Intelligence, vol. 2, no. 2-4, pp. 97-120, 2008.

[12] V. Gazi, "Swarms aggregation using artificial potentials and sliding mode control," IEEE Transactions on Robotics, vol. 21, no. 4, pp. 1208-1214, 2005.

[13] I. Škrjanc and G. Klančar, "Optimal cooperative collision avoidance between multiple robots based on bernstein-bézier curves," Robotics and Autonomous Systems, vol. 58, no. 1, pp. 1-9, 2010.

[14] E. Ferrante, A. E. Turgut, C. Huepe, A. Stranieri, C. Pinciroli, and M. Dorigo, "Self-organized flocking with a mobile robot swarm: a novel motion control method," Adaptive Behavior, p. 1059712312462248, 2012.

[15] R. Boque, "Miniature and microrobots: a review of recent developments," Industrial Robot: An International Journal, vol. 42, no. 2, pp. 98-102, 2015

[16] A. J. C. Sharkey, "Swarm robotics and minimalism," Connection Science, vol. 19, no. 3, pp. 245-260, 2007.

[17] A. Martinoli, "Collective complexity out of individual simplicity," Artificial Life, 2001.

[18] C. Moeslinger, T. Schmickl, and K. Crailsheim, "A minimalist flocking algorithm for swarm robots," in Advances in Artificial Life. Darwin Meets von Neumann. Springer, 2009, pp. 375-382.

[19] Y. Chen and T. C. Henderson, Experimental Robotics VII, ser. Lecture Notes in Control and Information Sciences. Springer, 2002, vol. 271, ch. S-NETS: Smart Sensor Networks, pp. 81-90. 
[20] L. Sabattini, C. Secchi, N. Chopra, and A. Gasparri, "Distributed control of multirobot systems with global connectivity maintenance," Robotics, IEEE Transactions on, vol. 29, no. 5, pp. 1326-1332, 2013.

[21] H. Oh, A. Ramezan Shiraz, and Y. Jin, "Morphogen diffusion algorithms for tracking and herding using a swarm of kilobots," Soft Computing, 2016, doi:10.1007/s00500-016-2182-2.

[22] A. F. Winfield and J. Nembrini, Morphogenetic Engineering. Springer, 2012, ch. Emergent swarm morphology control of wireless networked mobile robots, pp. 239-271.

[23] N. Bulusu, J. Heidemann, and D. Estrin, "Gps-less low-cost outdoor localization for very small devices," Personal Communications, IEEE, vol. 7, no. 5, pp. 28-34, 2000.

[24] D. Fox, W. Burgard, H. Kruppa, and S. Thrun, "A probabilistic approach to collaborative multi-robot localization," Autonomous Robots, vol. 8, no. 3, pp. 325-344, 2000.

[25] L. Doherty, K. S. Pister, and L. El Ghaoui, "Convex position estimation in wireless sensor networks," in INFOCOM 2001. Twentieth Annual Joint Conference of the IEEE Computer and Communications Societies. Proceedings. IEEE, vol. 3. IEEE, 2001, pp. 1655-1663.

[26] J. Bruck, J. Gao, and A. A. Jiang, "Localization and routing in sensor networks by local angle information," ACM Transactions on Sensor Networks (TOSN), vol. 5, no. 1, p. 7, 2009.

[27] N. Trawny and S. I. Roumeliotis, "On the global optimum of planar, range-based robot-to-robot relative pose estimation," in Robotics and Automation (ICRA), 2010 IEEE International Conference on. IEEE, 2010, pp. 3200-3206.

[28] D. Moore, J. Leonard, D. Rus, and S. Teller, "Robust distributed network localization with noisy range measurements," in Proceedings of the 2nd international conference on Embedded networked sensor systems, ACM. ACM, 2004, pp. 50-61.

[29] A. Cornejo and R. Nagpal, "Distributed range-based relative localization of robot swarms," in Algorithmic Foundations of Robotics XI. Springer, 2015, pp. 91-107.

[30] A. Ramezan Shirazi, H. Oh, and Y. Jin, "Morphogenetic selforganization of collective movement without directional sensing," in Advances in Autonomous Robotics Systems. Springer, 2014, pp. 139150.

[31] D.-T. Lee and A. K. Lin, "Generalized delaunay triangulation for planar graphs," Discrete \& Computational Geometry, vol. 1, no. 3, pp. 201217, 1986.

[32] A. F. Winfield and J. Nembrini, "Emergent swarm morphology control of wireless networked mobile robots," in Morphogenetic Engineering. Springer, 2012, pp. 239-271.

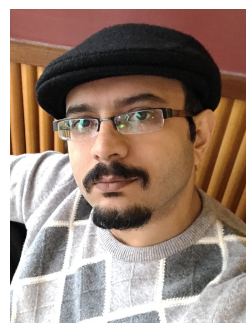

Ataollah Ramezan Shirazi received the BS degree in Solid State Physics from University of Tehran, Tehran, Iran, in 2008, and the MS degree in Mechatronics Engineering from University of Tabriz, Tabriz, Iran, in 2010. He worked as a Robotic Engineer at Ima Pardaz Tabriz Company and a Lecturer in Autonomous Systems at Islamic Azad University during 2010-2012. In 2013, he joined University of Surrey as a PhD candidate working on the Swarm-Organ project. Currently, he is a Research Fellow at the University of Hertfordshire on Agent-based Learning and Adaptation. His research interests cover collective behaviours, self-adaptive systems, self-organization, computational intelligence and cognitive robotics.

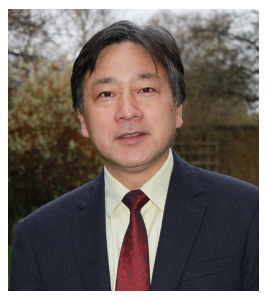

Yaochu Jin (M'98-SM'02-F'16) received the B.Sc., M.Sc., and Ph.D. degrees from Zhejiang University, Hangzhou, China, in 1988, 1991, and 1996 respectively, and the Dr.-Ing. degree from Ruhr University Bochum, Germany, in 2001.

$\mathrm{He}$ is a Professor in Computational Intelligence, Department of Computer Science, University of Surrey, Guildford, U.K., where he heads the Nature Inspired Computing and Engineering Group. He is also a Finland Distinguished Professor funded by the Finnish Agency for Innovation (Tekes) and a Changiiang Distinguished Visiting Professor appointed by the Ministry of Education, China. His research interests include evolutionary computation, neural networks, evolutionary developmental systems, multi-objective and secure machine learning, with their applications to complex systems design, swarm robotics and bioinformatics. He has (co)authored over 200 peerreviewed journal and conference papers and been granted eight patents on evolutionary optimization. He has delivered 25 invited keynote speeches at international conferences.

$\mathrm{He}$ is the Editor-in-Chief of the IEEE TRANSACTIONS ON COGNITIVE AND DEVELOPMENTAL SYSTEMS and Co-Editor-in-Chief of Complex \& Intelligent Systems. He is also an Associate Editor or Editorial Board Member of the IEEE TRANSACTIONS ON EVOLUTIONARY COMPUTATION, IEEE TRANSACTIONS ON CYBERNETICS, IEEE TRANSACTIONS ON NANOBIOSCIENCE, Evolutionary Computation, BioSystems, Soft Computing, and Natural Computing. Dr Jin is an IEEE Distinguished Lecturer (2017-2019) and was the Vice President for Technical Activities of the IEEE Computational Intelligence Society (2014-2015). He is the recipient of the 2017 IEEE Transactions on Evolutionary Computation Outstanding Paper Award, the CEC'2017 Best Student Paper Award, the 2014 and 2016 IEEE Computational Intelligence Magazine Outstanding Paper Award, the CIBCB'2010 Best Paper Award, and FOCI'2007 Best Student Paper Award. $\mathrm{He}$ is a Fellow of IEEE. 